\title{
Fair Efficient Call Admission Control Policies for Heterogeneous Traffic Streams in a Packet Routing Server Using The DTM Technology
}

\author{
Chih-Jen Chang ${ }^{1}$ and Arne A. Nilsson ${ }^{2}$ \\ ${ }^{1}$ Network Research Laboratory, Corporate Research Laboratories, Motorola Inc., \\ 20 Cabot Blvd., MS: M4-15, Mansfield ,MA 02048, USA \\ Chih-jen. Chang@motorola.com \\ ${ }^{2}$ Center for Advanced Computing and Communication, Department of Electrical \& \\ Computer Engineering, North Carolina State University, \\ Raleigh, NC 27695, USA \\ Nilssonencsu.edu
}

\begin{abstract}
The issue of fairness has new prominence in multi-service networks where the diverse service characteristics can result in a very unfair resource allocation unless the issue is considered explicitly at the design stage. In this paper, we study the fairness issue of a Packet Routing Server (PRS) architecture based on the Dynamic synchronous Transfer Mode (DTM) access technology. DTM provides a dynamic bandwidth allocation scheme to support various types of telecommunications applications. However, this flexibility also promotes within DTM a lack of fairness concerning medium utilization as no enforcement scheme has been implemented in the DTM access technology. We demonstrate the unfairness of the DTM access scheme and provide resource reservation schemes to ensure that each type of traffic has its relatively fair share of the medium. Our results show that the dynamic resource reservation achieves a better medium utilization and a fair access of the resource in the DTM PRS.
\end{abstract}

\section{Introduction}

As new and emerging multimedia telecommunication applications and other internet initiated activities have been developed and deployed over the past few years, the services provided by the traditional communication networks have also been expanded into a new dimension. First of all, the expansion of the network services makes the demands on bandwidth become less homogeneous. Also, some new multimedia applications such as voice and video may require special handling in order to maintain the quality of communications. Therefore, communication networks which provide integrated services, support for real-time applications, high speed access, and high bandwidths are needed in order to support the variety bandwidth demands of different applications. One such network based on the Dynamic synchronous Transfer Mode (DTM) [1], [2] access technology has been proposed. 
DTM is a shared medium broadband technology based on fast circuit switching [3] and dynamic TDM [4]. Because of its circuit switching nature, it guarantees each host a certain bandwidth or even uses a large fraction of the available bandwidth for effective data transmission. In addition, it provides support for the dynamic allocation of bandwidth such that the networks can adapt to variations in the traffic, and it divide its bandwidth between the hosts according to their demands. The PRS architecture we study in this paper is essentially a shared medium switching mechanism using the DTM access technology as shown in figure 1. The functionality of the PRS can be used as a bridge which connects the different Local Area Networks (LANs) or a router which connect LANs with the WANs [5]. Both implementations are to provide a new way of high speed access for internetworking. An example of the implementation of this PRS is shown in figure 2.

For those networks providing integrated services, the issue of fairness has received some attention in both circuit switched and packet switched networks. However, it has usually been considered a secondary issue as the primary concerns are often to maximize the network throughput in circuit switched networks and to minimize the average delay in packet switched networks. DTM is no exception to this rule, as it provides a mechanism for dynamic bandwidth allocation, but no mechanism to ensure the fair access to the medium. As in the integrated service environment, an ill-behaved user can easily occupy most of the resource if no enforcement has been implemented in the network.

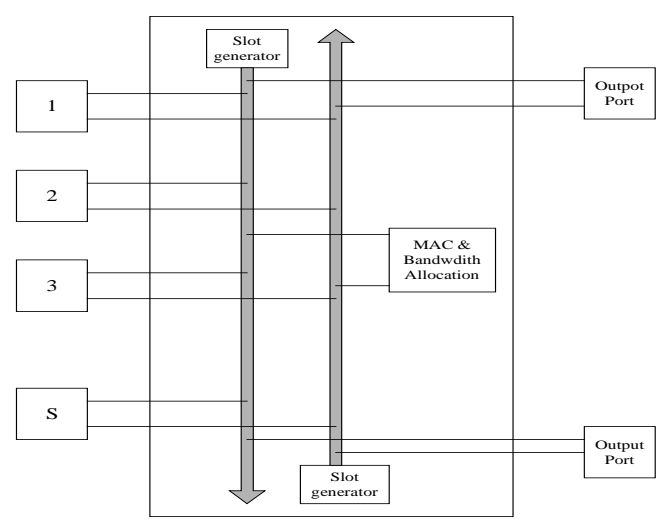

Fig. 1. A DTM Packet Routing Architecture

In this paper, we study the fairness issue for the call admission control schemes of a DTM PRS. The fairness issue is based on the utilization of the shared medium as a function of heterogeneous traffic streams. All of the performance comparisons are on the basis of the differing traffic types. We assume that the DTM PRS is basically a multi-service loss system, that is, a host which cannot successfully allocate sufficient bandwidth for data transfer will attempt to allocate the same amount of bandwidth after a random delay. The bandwidth we mentioned above is actually a corresponding 
set of 64-bit time slots in a DTM frame (where each frame has a transmission time of $125 \mu S$ ) and we assume a finite population of traffic sources.

This paper is organized as follows. In Section 2, we recall some basic concepts of call admission control policies which have been used in circuit switching networks and the analytical models for these call admission control policies under the finite population assumption. In Section 3, we demonstrate the unfairness issue in a DTM PRS and provide a solution to provide a fair access of the medium for this DTM PRS. In the final section, we provide a summary of the results in this paper.

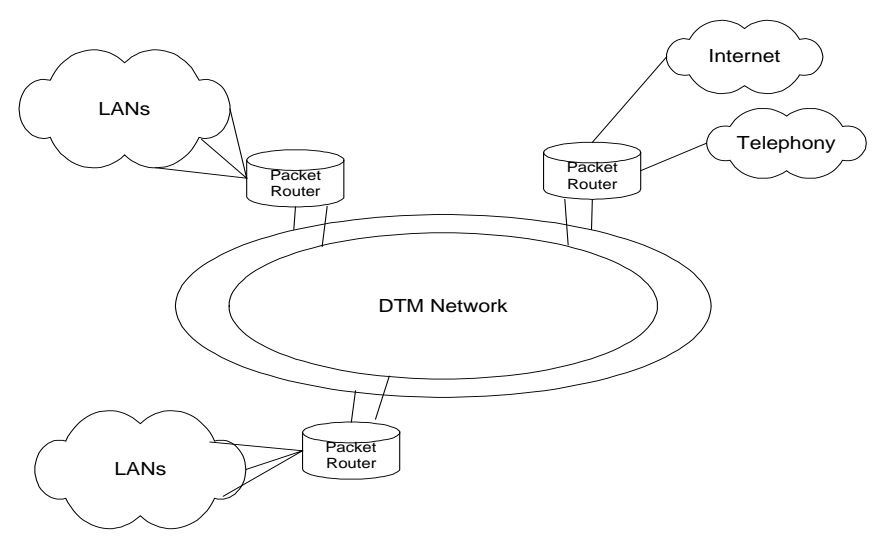

Fig. 2. Principal view of packet router solution for campus networks

\section{Call Admission Control Policies}

Typically, call admission control policies for circuit switched networks are based upon the derivation of the peak bandwidth of a given source. These policies can be divided into two categories: complete sharing and trunk reservation. In our study, we investigate the fairness issue for three different call admission control schemes originating from these two categories. We describe these schemes in the following sections.

\subsection{Complete Sharing Policy}

We consider a DTM PRS with a fixed bus capacity of $C$ Mbps. A number $R$ different traffic classes share the bus in a complete sharing mode as DTM is based on a fast circuit switching scheme and a channel is established for each call. Each type of traffic requires $b_{r}$ time slots as $r=1, \ldots, R$. There are finite number of traffic sources $S_{r}$, where $r$ varies from $l$ to $R$ for each class $r$. The arrival traffic of service class $r$ is assumed to follow an on-off process with the mean arrival rate $\beta_{r}$ per idle source, and mean holding time $1 / \mu_{r}$. It is assumed that both the idle time and the busy time 
of the on-off process have generally distributions. We let $C_{f}$ be the available capacity of the bus upon arrival of a burst of type $r$. The request will be blocked if $C_{f}<b_{r}$; otherwise, the request is granted. We will refer to this mode as call admission control without reservation.

The analytical model of this call admission control policy is based upon the Multirate Engset model [6], and it is known to have the feature of being insensitive to the inter-arrival and holding time distribution of the traffic sources [7]. Essentially, the steady state probabilities of this model have a product form solution [8]

$$
p\left(n_{1}, n_{2}, \ldots, n_{R}\right)=p(\underline{0}) \prod_{r=1}^{R}\left(\begin{array}{l}
S_{r} \\
n_{r}
\end{array}\right) \alpha_{r}^{n_{r}}
$$

where $n_{r}$ is the number of busy sources of class $r$ and $\alpha_{r}$ is defined as $\alpha_{r}=\frac{\beta_{r}}{\mu_{r}}$, the offered traffic per idle source of class $r$. Following the development presented by Kaufman [9] and Roberts [10], and expanding the dimensionality, a function is introduced in [8] which is defined as follows:

$$
q(\underline{S}, m)=\sum_{\sum_{r} n_{r} b_{r}=m} \prod_{r=1}^{R}\left(\begin{array}{l}
S_{r} \\
n_{r}
\end{array}\right) \alpha_{r}^{n_{r}}
$$

, where $\underline{S}=\left(S_{1}, S_{2}, \ldots, S_{R}\right)$. The variable $m$ has the obvious meaning of being the number of busy resources. The blocking probabilities for class $r\left(B_{r}\right)$ are actually the steady state probabilities when an arrival from class $r$ finds that $C-m<b_{r}$. Therefore,

$$
B_{r}=\frac{\sum_{j=C-b_{r}+1}^{C} q(\underline{S}, j)}{\sum_{i=0}^{C} q(\underline{S}, i)}
$$

As indicated in [8], there is a numerical instability with this formula. Therefore, in [8], yet another probabilistic interpretation is also introduced by defining a function as indicated in (4). This function represents the probability of having $m-k$ servers busy in a system with $m$ servers. We note that $0 \leq k \leq m$ and $\beta(\underline{S}, m, k)=0$ otherwise.

$$
\beta(\underline{S}, m, k)=\frac{q(\underline{S}, m-k)}{\sum_{i=0}^{m} q(\underline{S}, i)}
$$

Recursive equations for the function $\beta(\underline{S}, m, k)$ were also developed in [8] by using binomial identities as follows: 


$$
q(\underline{S}, m)=\sum_{i_{R}=0}^{1} \ldots \sum_{i_{1}=0}^{1}\left(\prod_{r=1}^{R} \alpha_{r}^{n_{r}}\right) q\left(\underline{S}-\underline{1}, m-\sum_{r=1}^{R} i_{r} b_{r}\right)
$$

In this paper, we simplify the derivation from that of the original paper. First, we rewrite the $\beta$-function as follows:

$$
\beta(\underline{S}, m, k)=\frac{q(\underline{S}, m-k)}{\sum_{i=0}^{m-1} q(\underline{S}, i)+q(\underline{S}, m)}
$$

Following the binomial identities, the first term in the denominator becomes:

$$
\sum_{i=0}^{m-1} q(\underline{S}, i)=\sum_{i=0}^{m-1} \sum_{i_{1}=0}^{1} \sum_{i_{2}=0}^{1} \ldots \sum_{i_{R}=0}^{1}\left(\prod_{r=1}^{R} \alpha_{r}^{i_{r}}\right) q\left(\underline{S}-\underline{1}, i-\sum_{r=1}^{R} i_{r} b_{r}\right)
$$

, and the second term in the denominator is then

$$
q(\underline{S}, m)=\frac{1}{m} \sum_{r=1}^{R} S_{r} \alpha_{r} b_{r} \sum_{\substack{i_{l}=0, l \neq r \\ l=1,2, \ldots, R}}^{1}\left(\prod_{\substack{j=1 \\ j \neq r}}^{R} \alpha_{j}^{i_{j}}\right) q\left(\underline{S}-\underline{1}, m-b_{r}-\sum_{\substack{j=1 \\ j \neq r}}^{R} i_{j} b_{j}\right)
$$

and the numerator is:

$$
q(\underline{S}, m-k)=\sum_{i_{1}=0}^{1} \sum_{i_{2}=0}^{1} \ldots \sum_{i_{R}=0}^{1}\left(\prod_{r=1}^{R} \alpha_{r}^{i_{r}}\right) q\left(\underline{S}-\underline{1}, m-1-\left(k+\sum_{r=1}^{R} i_{r} b_{r}-1\right)\right)
$$

Divided both the numerator and denominator by $\sum_{i=0}^{m-1} q(\underline{S}-\underline{1}, i)$, and let $\beta(\underline{S}, m, k)=\frac{\text { Numerator }}{\text { Denominator }}$. Thus, the Numerator takes on the form:

$$
\sum_{i_{1}=0}^{1} \sum_{i_{2}=0}^{1} \ldots \sum_{i_{R}=0}^{1}\left(\prod_{r=1}^{R} \alpha_{r}^{i_{r}}\right) \beta\left(\underline{S}-\underline{1}, m-1, k+\sum_{r=1}^{R} i_{r} b_{r}-1\right)
$$

, and the Denominator is:

$$
\begin{aligned}
& \sum_{i=0}^{m-1} \sum_{i_{1}=0}^{1} \sum_{i_{2}=0}^{1} \ldots \sum_{i_{R}=0}^{1}\left(\prod_{r=1}^{R} \alpha_{r}^{i_{r}}\right) \beta\left(\underline{S}-1, m-1, i-\sum_{r=1}^{R} i_{r} b_{r}\right)+ \\
& \frac{1}{m} \sum_{r=1}^{R} S_{r} \alpha_{r} b_{r} \sum_{\substack{i_{l}=0, l \neq r \\
l=1,2, \ldots, R}}^{1}\left(\prod_{\substack{j=1 \\
j \neq r}}^{R} \alpha_{j}^{i_{j}}\right) \beta\left(\underline{S}-\underline{1}, m-1, b_{r}-1+\sum_{\substack{j=1 \\
j \neq r}}^{R} i_{j} b_{j}\right)
\end{aligned}
$$

The blocking probabilities can now be computed by using the following equation: 


$$
B_{r}=\sum_{k=0}^{b_{r}-1} \beta(\underline{S}, C, k)
$$

\subsection{Restricted Resource Reservation Policy}

Normally a call with higher bandwidth requirement will be blocked with larger probability. To maintain the grade of service for individual classes in a system with heterogeneous traffic streams, a resource reservation mechanism is often used. In this section, we consider the same system as in the previous section with a reservation scheme. In the reservation mechanism, we denote the reservation threshold assigned to class $r$ by $T_{r}, r=1,2, \ldots, R$. For those classes which are able to use the reserved resources, no threshold is assigned. We assume that the set of those classes with threshold assigned is denoted as $A$. The algorithm works as illustrated in figure 3 .

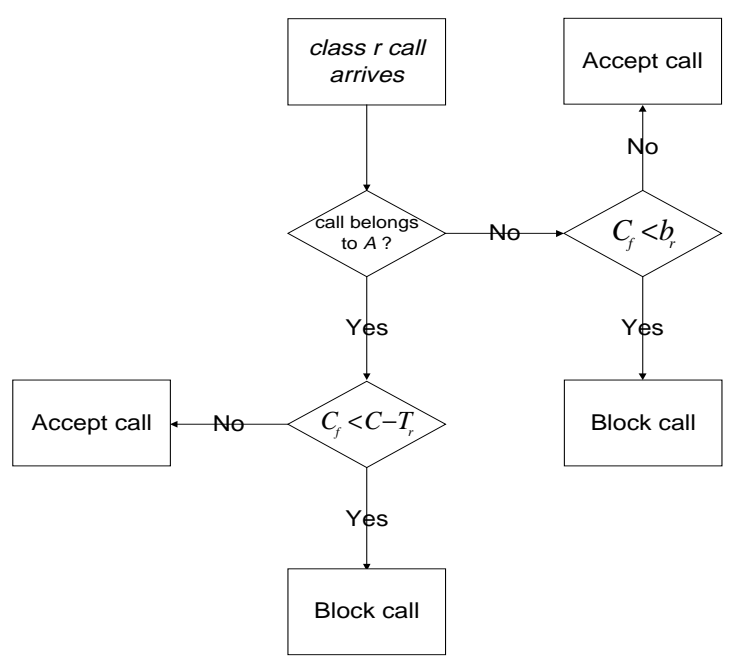

Fig. 3. Restrict Resource Reservation Scheme

As discussed in [11], the general rule for balancing the blocking probabilities of calls from different classes $i, j, k, \ldots$ is to set the corresponding thresholds $T_{i}, T_{j}, T_{k}, \ldots=C-\max \left\{b_{i}, b_{j}, b_{k}, \ldots\right\}$. This rule will equalize the blocking probabilities of traffic types $i, j, k, \ldots$ in the case of infinite population.

In the case of trunk reservation in systems with finite population, we consider the Markov chains where each state represents the number of total busy time slots. Because of the reservation mechanism, the states from $\left(C-b_{R}+1\right)$ to $C$ in the Markov 
chains do not have transitions to the states within this subset, making the Markov chains irreversible. Hence, product form solutions is no longer valid in this case. However, in principle, we can still obtain a numerical approximation by using the recursive algorithm we introduced in the previous section. Basically, the analysis is to follow the $\beta(\underline{S}, C, k)$ recursion, however, some modifications need to be made in order to obtain the numerical approximation.

We let $\beta(\underline{S}, m, k)=\frac{\text { Numerator }}{\text { Denominator }}$, the modification is:

Numerator $=\sum_{i_{1}=0}^{1} \sum_{i_{2}=0}^{1} \ldots \sum_{i_{R}=0}^{1}\left(\prod_{r=1}^{R} \alpha_{r}^{i_{r}}\right) \beta\left(\underline{S}-\underline{1}, m-1, k+\sum_{r=1}^{R} i_{r} b_{r}-1\right)$

, and the Denominator will have the form in equation (15). We note that:

$$
\begin{gathered}
b_{r}(m-k)=\left\{\begin{array}{c}
b_{r}: m-k \leq T+b_{r} \\
0: m-k>T+b_{r}
\end{array}, r=1,2, \ldots, R \text { and } T=C-\max \left(b_{1}, b_{2}, \ldots, b_{r}\right) .\right. \\
\sum_{i=0}^{m-1} \sum_{i_{1}=0}^{1} \sum_{i_{2}=0}^{1} \ldots \sum_{i_{R}=0}^{1}\left(\prod_{r=1}^{R} \alpha_{r}^{i_{r}}\right) \beta\left(\underline{S}-1, m-1, i-\sum_{r=1}^{R} i_{r} b_{r}\right)+ \\
\frac{1}{m} \sum_{r=1}^{R} S_{r} \alpha_{r} b_{r}(m-k) \sum_{\substack{i_{l}=0, l \neq r \\
l=1,2, \ldots, R}}^{1}\left(\prod_{\substack{j=1 \\
j \neq r}}^{R} \alpha_{j}^{i_{j}}\right) \beta\left(\underline{S}-\underline{1}, m-1, b_{r}-1+\sum_{\substack{j=1 \\
j \neq r}}^{R} i_{j} b_{j}\right)
\end{gathered}
$$

Again, the blocking probabilities for class $i$ is:

$$
B_{r}=\sum_{k=0}^{\max \left\{b_{r}-1, T-1\right\}} \beta(\underline{S}, C, k)
$$

\subsection{Dynamic Resource Reservation Policy}

Although the restricted resource reservation policy can balance the blocking probabilities for different traffic classes, the total medium utilization decreases. However, our goal is not only to provide a technique for fair access of the medium but also to maintain a better medium utilization. Therefore, we introduce the dynamic reservation policy in this section. This mechanism relies on the number of free slots upon the arrival of a call from class $r$. We assign the threshold of the system the same as the restricted resource reservation as we wanted to balance the blocking probabilities for different traffic classes. We consider a PRS with $R$ traffic classes and each require $b_{r}$ time slots for transmission. It is also assumed that the number of required time slots for each class have the following relationship: $b_{1} \leq b_{2} \leq \ldots \leq b_{R}$. Thus, we assign the threshold $T=C-\max \left\{b_{1}, \ldots, b_{R}\right\}$. Obviously, $T$ equals to $C-b_{R}$ in this case. The call admission algorithm works as illustrated in figure 4 . As shown in figure 4 , it may 
seem that the system still admits calls with lower bit rates when the threshold has been reached or exceeded, thus introducing unfairness to classes with higher bit rates. The argument is that since the number of reserved time slots are not always equal to $b_{R}$, in the case of $C_{f}<b_{R}$, the resources are wasted and the unfairness still exists under restrict reservation scheme in a system with finite population. The mechanism shown in figure 4 admits calls from a certain class strictly based on the current available resources when the threshold has been reached or exceeded, hence providing higher utilization and better fairness in a systems with finite population. We will show the simulation results of using this reservation mechanism in next section.

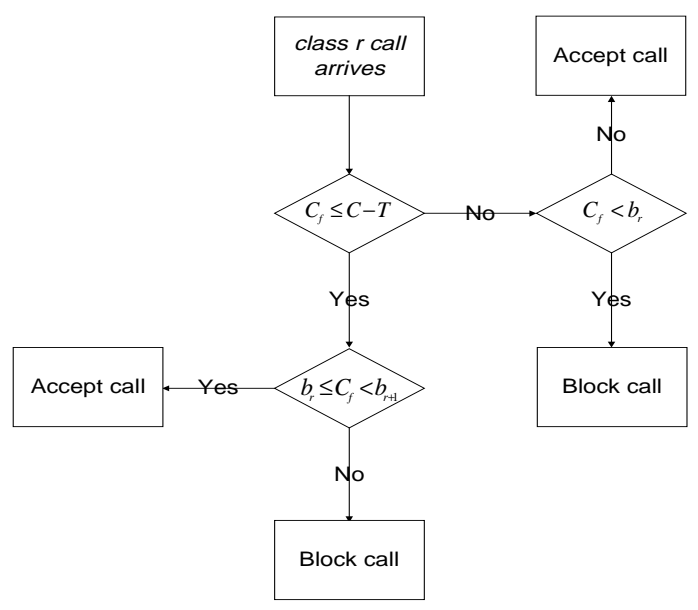

Fig. 4. Dynamic Resource Reservation Scheme.

We note that due to the complexity of this algorithm, only simulation results are shown in this paper.

\section{Numerical Results}

First of all, we would like to point out that our previous work [12], [13] has shown that Multi-rate Engset model is a valid model for the DTM PRS architecture. Therefore, we only present the analytical results for the call admission control policy without reservation in this paper. We demonstrated the unfairness of medium access in a DTM PRS. The system has two dual fiber bus, each with the capacity of $155 \mathrm{Mbps}$. We increase the number of traffic sources attached to the PRS thus increasing the offered load to the fiber bus. The offered load is the normalized bit rate traffic for each class and is computed by using: Offered $\operatorname{Load}=\frac{S_{r} \alpha_{r}}{1+\alpha_{r}\left(1-B_{r}\right)} * \frac{b_{r}}{C}$, where $S_{r}$ 
represent the number of traffic sources from class $r$, and $\alpha_{r}, b_{r}, B_{r}$ are the same as defined in section 2.1. $C$ is the capacity of the fiber bus.

We consider a system with three traffic classes as shown in figures 5 and 6 and assume $\alpha_{1}=1, \alpha_{2}=1 / 3$ and $\alpha_{3}=1 / 7$. It is also assumed that class 1 requires bandwidth of $3.072 \mathrm{Mbps}$, class 2 requires bandwidth of $6.144 \mathrm{Mbps}$ and class 3 requires bandwidth of $12.288 \mathrm{Mbps}$.

Both figures 5 and 6 show that the traffic classes with higher bit rates suffers from higher blocking probabilities and lower throughput at the same value of offered traffic. These indicate the unfairness in the complete sharing scheme.

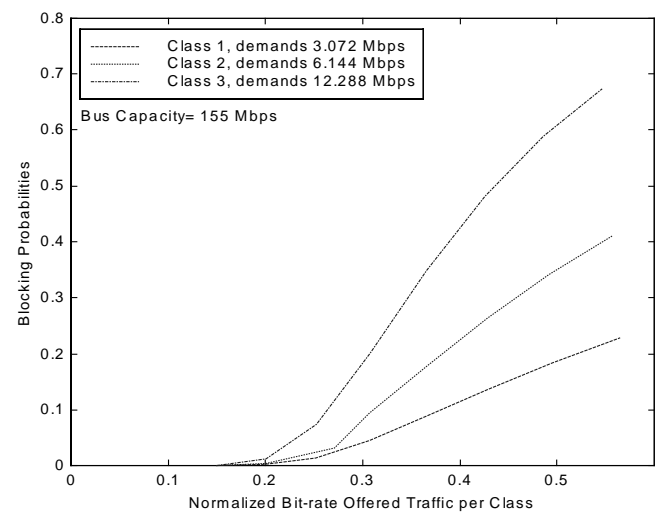

Fig. 5. Blocking probabilities for three traffic classes in a DTM PRS without resource reservation.

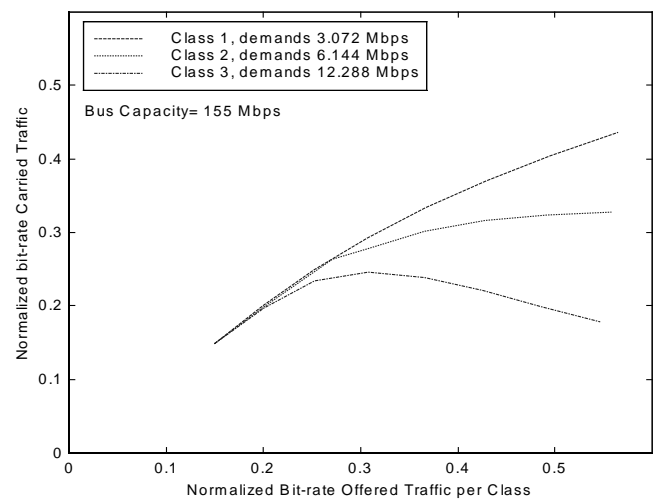

Fig. 6. Normalized carried traffic for three traffic classes in a DTM PRS without resource reservation.

We then consider the same system configuration with restricted resource reservation scheme and show the results in figures 7 and 8. As shown in figure 7, the approximation of the blocking probabilities obtained from the recursive formula are close to the simulation results in this system. We also show in figures 7 that the blocking probabilities for different traffic classes are brought closer by employing the 
resource reservation policy. However, as indicated in figures 7 and 8, under higher load, this reservation mechanism still suffers from unfairness problem.

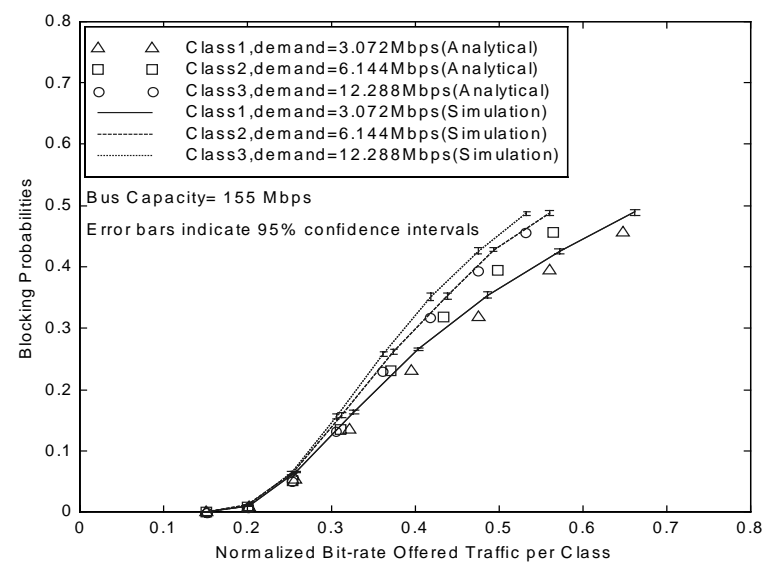

Fig. 7. Blocking probabilities for three traffic classes in a DTM PRS with restricted resource reservation.

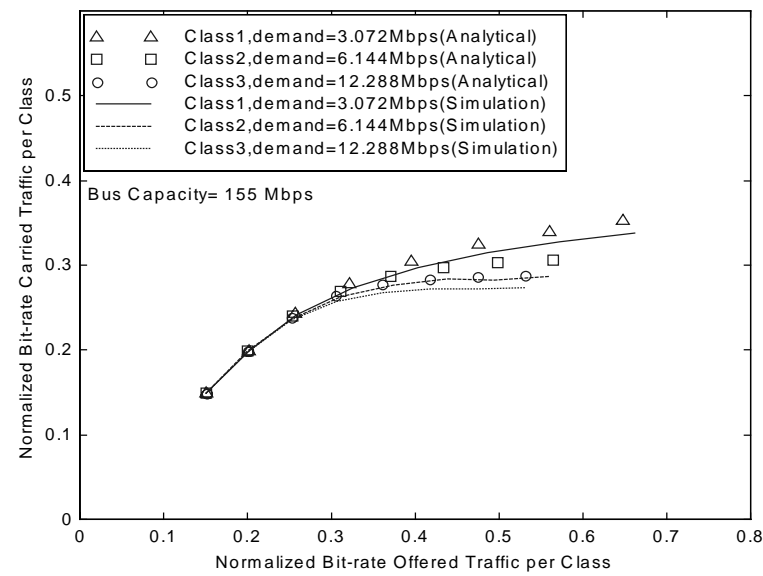

Fig. 8. Normalized carried traffic for three traffic classes in a DTM PRS with restricted resource reservation.

Finally, we consider the same system again, however, with dynamic resource reservation scheme. We show the results in figures 9 and 10. Figure 9 shows that the curves of blocking probabilities for different traffic classes are closer with the dynamic reservation mechanism than with the restricted reservation scheme. We also present in figure 10 that the throughputs for different classes are closer under the dynamic reservation technique. Thus, we have achieved a fair medium access and better utilization for heterogeneous traffic streams by employing the dynamic reservation mechanism into the DTM PRS architecture. 


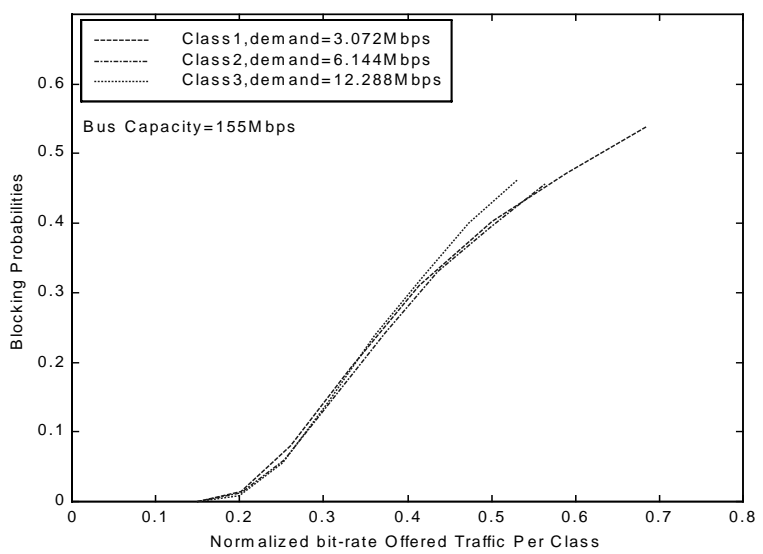

Fig. 9. Blocking probabilities for three traffic classes in a DTM PRS with dynamic resource reservation.

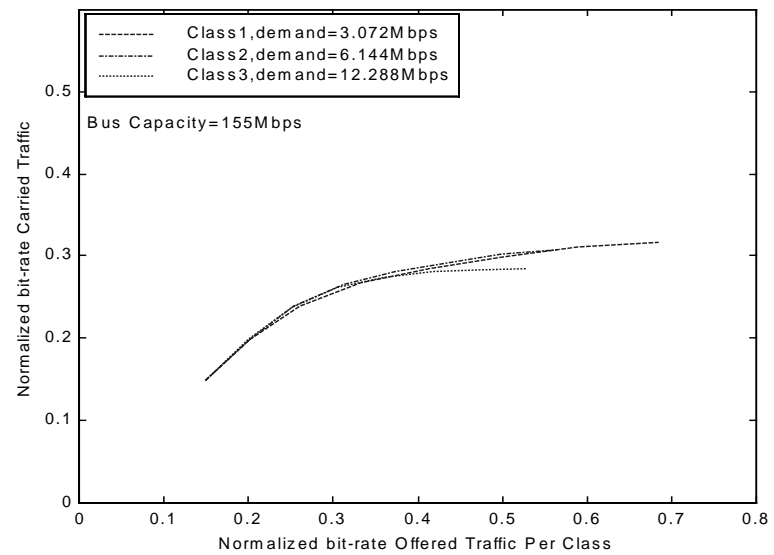

Fig. 10. Normalized carried traffic for three traffic classes in a DTM PRS with dynamic resource reservation.

\section{Conclusion}

In this paper, we have considered a DTM PRS architecture with different types of services. We investigate the fairness issue in this system by employing different call admission control policies including complete sharing, restrict reservation and dynamic reservation. The analytical models and computation schemes addressing the related call admission control policies have been reviewed except for the dynamic reservation due to its complexity. Simulation results have been carried out to illustrate the efficiency and grade of service management capability of resource reservation 
mechanisms. In conclusion, the dynamic reservation policy provides a better fairness and medium utilization for the DTM PRS system than the other call admission control policies.

\section{References}

1. Lars H. Ramfelt, “Architecture for High Performance Optical Networks”, Doctor of Technology Dissertation, Department of Teleinformatics, The Royal Institute of Technology, Sweden, February 1996.

2. Christer Bohm, "The DTM Protocol - Design and Implementation", Licentiate Thesis, Department of Teleinformatics, The Royal Institute of Technology, Sweden, February 1994.

3. Per Lindgren, "A Multi-Channel Network Architecture Based on Fast Circuit Switching", Doctor of Technology Dissertation, Department of Teleinformatics, The Royal Institute of Technology, Sweden, May 1996.

4. L.T. Wu, S.H. Lee and T.T. Lee, "Dynamic TDM- A Packet Approach to Broadband Networking", Proceedings of IEEE Globalcom, Page 1585-1592, 1987.

5. Markus Hidell, "Supporting the Internet Protocols in a High Performance Real-Time Network", Licentiate Thesis, KTH, Stockholm, April, 1996.

6. J.W. Cohen, "The generalized Engset formulae”, Philips Telecomm., 18 (1957), pp 150-170.

7. Ryszard Syski, "Introduction to Congestion Theory in Telephone Systems", Elsevier Science, 1986.

8. Mark J. Perry and Arne A. Nilsson, Multirate Blocking Probabilities: "Numerically Stable Computations", ITC15, pp. 1359 - 1368, June 1997.

9. J.S. Kaufman, "Blocking in a Shared Resource Environment", IEEE Trans. On Communication, Vol. 29, October 1981, pp. $1474-1481$

10. J.W. Roberts, "Teletraffic Models for the Telecom 1 Integrated Services Network", ITC 10, Montreal 1983, paper 1.1.2.

11. P. Tran-Gia and F. Hübner, "An Analysis of Trunk Reservation and Grade of Service Balancing Mechanisms in Multiservice Broad Networks", IFIP Transactions C-15, 1993, pp. 83- 97.

12. C.J. Chang and A.A. Nilsson, "Performance Evaluation of DTM Access Nodes", TR98/05, Center for Advanced Computing and Communications, North Carolina State University, April 1998

13. C.J. Chang and A.A. Nilsson, "Analytical Model for DTM Access Nodes”, TR98/11, Center for Advanced Computing and Communications, North Carolina State University, April 1998 